\title{
Early Prevention of Instability-Use of Self Propagating Graph for the Fast Search for Optimal Grid Nodes to Apply Countermeasures
}

Dmitrova, Evgenia; Jóhannsson, Hjörtur; Nielsen, Arne Hejde

Published in:

Proceedings of the 2013 IEEE PowerTech Conference

Link to article, DOI:

10.1109/PTC.2013.6652379

Publication date:

2013

Document Version

Early version, also known as pre-print

Link back to DTU Orbit

Citation (APA):

Dmitrova, E., Jóhannsson, H., \& Nielsen, A. H. (2013). Early Prevention of Instability-Use of Self Propagating Graph for the Fast Search for Optimal Grid Nodes to Apply Countermeasures. In Proceedings of the 2013 IEEE PowerTech Conference IEEE. https://doi.org/10.1109/PTC.2013.6652379

\section{General rights}

Copyright and moral rights for the publications made accessible in the public portal are retained by the authors and/or other copyright owners and it is a condition of accessing publications that users recognise and abide by the legal requirements associated with these rights.

- Users may download and print one copy of any publication from the public portal for the purpose of private study or research.

- You may not further distribute the material or use it for any profit-making activity or commercial gain

- You may freely distribute the URL identifying the publication in the public portal 


\title{
Early Prevention of Instability-Use of Self Propagating Graph for the Fast Search for Optimal Grid Nodes to Apply Countermeasures
}

\author{
Evgenia Dmitrova, Hjörtur Jóhannsson, Arne Hejde Nielsen \\ Elektro, Technical University of Denmark \\ Center for Electric Power and Energy \\ Kgs. Lyngby, Denmark \\ ed@elektro.dtu.dk, hj@elektro.dtu.dk, ahn@elektro.dtu.dk
}

\begin{abstract}
This paper presents a method for a fast determination of the grid nodes where countermeasures, in the form of changes in nodal admittance, would provide greatest impact on the stability margin for a specific generator that is facing the risk of instability. The sensitivity of the stability criteria for aperiodic small signal angular stability to the change in nodal admittance is used as a factor quantifying impact that the node has on the stability of a critical generator. In order to lower the number of nodes which are processed through sensitivity analysis, a selfpropagating graph with discrete steps is applied. The suggested method is tested on the IEEE 30 bus test system and on the 1648 bus US west coast test system where the results show that the number of nodes processed through sensitivity analysis are well reduced compared to the full sensitivity analysis, illustrating the potential of the developed approach for the fast identification of the optimal nodes for countermeasure application.
\end{abstract}

Index Terms- graph theory, power systems, sensitivity analysis, stability.

\section{INTRODUCTION}

The gradual integration of phasor measurement units into real power systems opens up new possibilities for modern control methods, including adaptive preventive control [1-2]. The ability to keep hand on power system pulse is a great evolution in the field of situational awareness. Clear understanding of the actual operational conditions allows increasing efficiency of power systems, specifically in terms of transmission system loading. Conventionally, the limitation on power capacitance of a certain transfer pass was defined by stability boundaries, pre-defined on the basis of system statistical data and off-line simulations. Additionally, a certain safety margin was used as a buffer to ensure stable operation in case if actual operational conditions would be worse than pre-modeled ones. Thus, the worst case scenario has been considered as a benchmark for power transfer limits setting; however actual operational conditions might vary towards a "lighter" case compared to modeled ones. Knowing the actual stability boundaries would help improve the efficiency and at the same time reliability of power systems. Taking advantage of the phasor measurements, future perspectives are to obtain complete observability of a grid, which motivates research and development of new methods for the real-time systems stability monitoring. Among others, the method allowing detection of Aperiodic Small Signal Angular Stability (ASSAS) problems at the early stage of their emergence, utilizing wide area measurements, have been introduced in [34]. The method, which is based on the analytically derived expression for a stability boundary [3], gave inspiration for the development of the early prevention method. In this method, the warning message about approaching stability boundaries is used as a triggering signal for the search of appropriate countermeasures. The main functionality of the early prevention method is the fast identification of the optimal countermeasure, taking into account the actual allocation and availability of control reserves in the grid, which insures instability avoidance. In order to optimize the countermeasure, the nodes which have the highest potential to influence stability of the critical generator should be found. As a decision regarding the application of the countermeasure may be time restricted, the number of nodes which might be considered as control candidates should possibly be reduced, skipping those nodes in the analysis, which a priory cannot help much to improve a critical situation. The method for identification of the optimal nodes for countermeasure application was presented in [5] and utilizes sensitivity analysis for quantification of the impact which a node has on the stability of a critical generator. This paper is focused on the method allowing considerable reduction of the time needed for the search of the optimal node in the grid to which a countermeasure should be applied.

The paper is organized as follows: Section II contains the theoretical description of the proposed approach for a selfpropagating graph; in Section III, illustration of the developed method applied to the IEEE30 bus test system and the 1648 bus US west coast test system is provided; Section IV contains the conclusion and perspectives for further research. 
Notation: Uppercase bold letters refer to matrixes. Complex numbers are underlined, * - denotes complex conjugate.

\section{METHODOLOGY}

\section{A. Optimal node for the preventive countermeasure application}

For the search of the optimal node for which to apply a countermeasure when a given generator is facing the risk of ASSAS loss, the stability criterion derived in [4] is utilized. The criterion is formulated for the two bus system representation, where one bus is representing the critical generator under consideration and the rest of the system is reduced to the Thevenin equivalent (Fig.1).

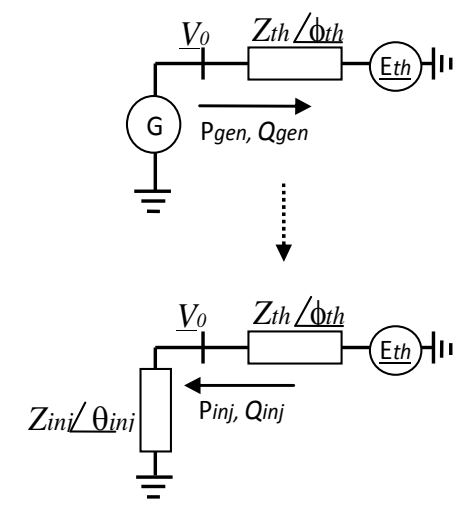

Figure 1. Equivalent system representation regarding critical generator.

The mathematical expression for the ASSAS criterion is shown in (1):

$$
\begin{cases}Z_{i n j}>=-Z_{t h} \cdot \sin \theta i n j / \sin \phi t h & - \text { stable operation } \\ Z_{i n j}<-Z_{t h} \cdot \sin \theta i n j / \sin \phi t h & \text {-unstable operation }\end{cases}
$$

Where Zinj corresponds to the magnitude of the generators' injection impedance, measured at the node of constant steady state voltage magnitude $\mathrm{V}_{0}, \mathrm{Z}_{\text {th }}$ is Thevenin equivalent of the system seen from the node of power injection. Oinj and $\phi$ th are the angles of $Z_{i n j}$ and $Z$ th respectively. The value of injection impedance is determined as follows:

$$
\underline{Z}_{i n j}=-\underline{V}_{0}^{2} /\left(P_{g e n}+j \cdot Q_{g e n}\right) *=\underline{V}_{0}^{2} / \underline{S}^{*}{ }_{i n j}
$$

Where $\mathrm{P}_{\text {gen }}, \mathrm{Q}_{\text {gen }}$ are an active and reactive power of a generator fed to a grid from the node of constant steady state voltage magnitude and $\underline{S}_{i n j}$ is the corresponding injection power which is equal to $\underline{\mathrm{S}}_{g e n}$ with opposite sign.

It was shown in [5] that in order to identify the nodes, which have high effectiveness for countermeasure application, meaning that the variation of admittance in those nodes has considerable impact on the stability of a given generator, it is enough to find out which nodes are most influencing the Thevenin impedance seen by this generator. For this purpose the sensitivity of $\mathrm{K}_{t h}=\mathrm{Z}$ th/sin $\phi$ th (1) to the variation of nodal admittance in each of the load nodes (nodes with neither generators nor constant voltage sources) should be calculated.
For this study the rescheduling of the generators is assumed to be not available, which reflects the case of full loading operation; thus only load nodes are considered as a target for countermeasure application. To calculate the sensitivity of the Thevenin impedance, seen by a given generator, to the nodal admittance variation the same change in admittance $\Delta \mathrm{Y}$ is applied in each load node in sequence, which implicitly represents the application of the countermeasure. Thus, for the given generator $k$ the following sensitivity is calculated (3) for each of the load nodes, forming the vector of sensitivities established in (4), where CEF stands for Control Efficiency Factor:

$$
S_{K t h_{k m}}=\left[\frac{\partial\left(Z_{t h} / \sin \left(\varphi_{t h}\right)\right)_{k}}{\partial\left(\underline{Y}_{m, m}\right)}\right]=\left[\frac{\partial\left(K_{t h_{k}}\right)}{\partial\left(\underline{Y}_{m, m}\right)}\right]
$$

Where $\mathrm{m}=1$..M, $\mathrm{M}$ - number of load nodes in the grid

$$
[C E F]_{k}=\left(S_{K t h_{k 1}} \ldots S_{K t h_{k m}} \ldots S_{K t h_{k M}}\right)
$$

When normalized, the elements of the CEF matrix are representing the relative efficiency of a countermeasure application to the corresponding node.

The number of load nodes in the real system reaches thousands; calculation of sensitivities $S_{K t h}$ for all of them in order to define which would be the optimal one for countermeasure application would require large computational power, which is a crucial issue, especially if close to real-time sensitivity analysis (SA) is desired. At the same time the number of nodes, which actually have considerable impact on the stability of a certain generator, is noticeably lower than the number of load nodes in the grid; thus there is an interest in developing an algorithm allowing reduction of the number of nodes processed through SA, initially sorting out the nodes which a priori do not have high CEF.

\section{B. Self propagating graph utilisation for limiting the amount of nodes processed through sensitivity analysis}

In order to limit the number of nodes which are to be processed through SA, it is necessary to develop an approach enabling nodes assessment in a way that those nodes having very low impact on the stability of a given generator can be sorted out early on.

It was noticed that with the increase of topological distance between the considered node and the critical generator, the value of sensitivity $S_{K t h_{k m}}$ tends to decrease. Depending on how meshed the grid is and what are the impedances of branches connecting the nodes the decrease of $S_{K t h_{k m}}$ happens unevenly, and in fact is defined by equivalent transport admittance $\mathrm{Y}_{k m}$ between the node and generator. However, in order to avoid the calculation of equivalent transfer admittance, the topological distance between the considered node and given generator was chosen as a reference criterion. As the main purpose of this study is to find the way allowing sorting out the nodes in which sensitivity $S_{K t h_{k m}}$ is below certain minimum level, let us denote for 
certainty the minimum acceptable sensitivity is $S_{K t h}$ min $S_{K t h}{ }_{\text {min }}$ is the criterion which would vary depending on topology of the grid. In meshed grids the sensitivity $S_{K t h_{k m}}$ decreases faster, which means that the minimum acceptable value for $S_{K t h}$ can be lowered in this case to ensure that a sufficient amount of load nodes capable of providing positive effect on generator's stability are considered. Furthermore, the sufficient number of nodes processed through SA, is dependent on the availability of the control reserves in the nodes with high sensitivity and generally might vary depending on the current control reserves allocation.

As there is a correlation between the increase of topological distance and the sensitivity decrease, the natural decision is to split nodes per sets with accordance to their topological distance to the critical generator. The topological distance in this context is the number of vertexes on the shortest path between the critical generator and given node, when the grid is represented as a graph; moreover, the edges in this graph are weightless. In order to organize the split of the nodes per sets, the concept of the self-propagating graph is used. The term "self-propagating graph" is reflecting the principle of discreet graphing where the nodes are added to the graph stepwise with respect to the length of the shortest path from the critical generator to the given node. On each step the set of equally topologically distant nodes is formed, each next set will contain the nodes which shortest path to the critical generator is one vertex more, comparing to the previous set. As soon as a new set of nodes is added to the graph, the sensitivity $S_{K t h}$ should be calculated for each of the nodes in the set. If the sensitivity in the node under consideration appears to be below the preset value $S_{K t h}{ }_{\min }$, the node is marked as the dead-end node and further development of the graph through this node is blocked. Thus when the next set is formed, the path of the considered nodes cannot lay through the dead-end node.

In order to keep track of the visited nodes, which have been already included in the self-propagating graph, the nodes from the considered set are added to the list $\mathbf{L}$, on each step of the propagation; before adding a node to the next set the check is carried out. Further efficiency improvement of the suggested algorithm can be achieved if the graphing can be organized in a way so that the resulting graph is a tree. By eliminating loops in the graph the double check of sensitivity at the same node can be avoided. Looping can take place in the created graph when a given node has more than one path with the same number of vertexes on the way to the beginning of the graph. This problem is avoided by utilizing first-in-first-out (FIFO) [6] queuing when forming the set. The check and update of the list $\mathbf{L}$ happens each time when the node is added to the queue. If the node is not listed in $\mathbf{L}$ it gets added to the self-propagating graph, at the same time $\mathbf{L}$ is updated by including this node to the list of visited nodes. This will allow keeping track of the visited nodes, not only when comparing a set of nodes to the previous set, but within a set as well. If those principles of graphing are followed, the resulting graph is a tree and all nodes added to the graph are visited only ones.

The propagation of the graph continues until either all load nodes in the grid were visited and sensitivity $S_{K t h_{k m}}$ has been calculated for all of them, or further propagation of the graph is not possible as all the vertexes in the last added set were marked as a dead-end vertexes. Satisfying of the second condition for interruption of graph propagating means that the CEF for any other node, not included in the created graph, will be below $S_{K t_{\text {min }}}$ and those nodes should not be participating in SA. Thus the number of nodes which are included in the sensitivity analysis might be considerably reduced, which leads to noticeable improvement of computational time. The validity of the suggested approach was tested on the IEEE 30 bus test system and on the 1648 bus US west coast test system, and the results obtained are presented in the next section of this paper.

\section{RESUlTS AND DISCUSSIONS}

\section{A. Self-propagating graph method applied to the IEEE 30 bus test system}

Fig. 2 shows the IEEE 30-bus test system diagram, with the system data borrowed from the power system test archive [7]. Few modifications were done to the system: synchronous compensators in the grid were replaced by generators and three-winding transformers were replaced by two twowinding transformers. The terminal of generator G1 is chosen as a reference bus.

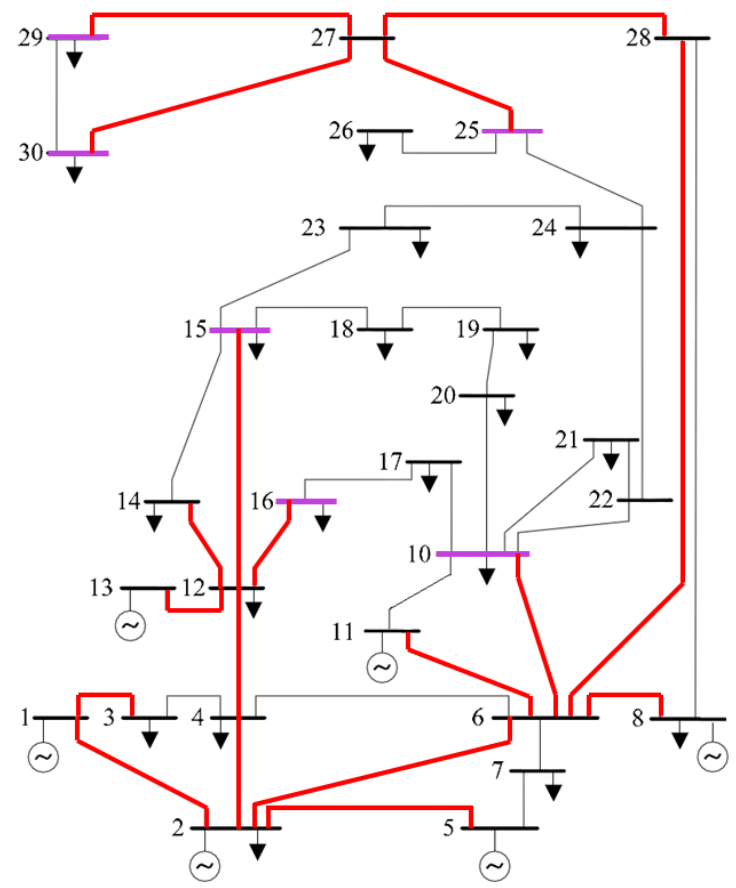

Figure 2. Single line diagram of IEEE 30 bus test system; red color is marking edges of resulting self-propagating graph, purple is marking deadend vertexes. 
For the demonstration of the method described in Section II of this paper generator $G_{1}$ was picked as a unit regarding which the SA is to be carried out. For the given study case the change in reactive component of shunt admittance equaled to $\Delta \mathrm{Y}_{\mathrm{m}, \mathrm{m}}=0.01$ p.u. was applied as a nodal admittance variation. The minimal sensitivity down to which the impact of the node to the stability of $\mathrm{G}_{1}$ is considered noticeable was chosen to be equal to 0.3333 , which is one third of the maximal sensitivity when CEF is normalized. Fig. 3 shows the list of sets formed according to the shortest topology distance to the source vertex, which is $\mathrm{G}_{1}$ in this case.

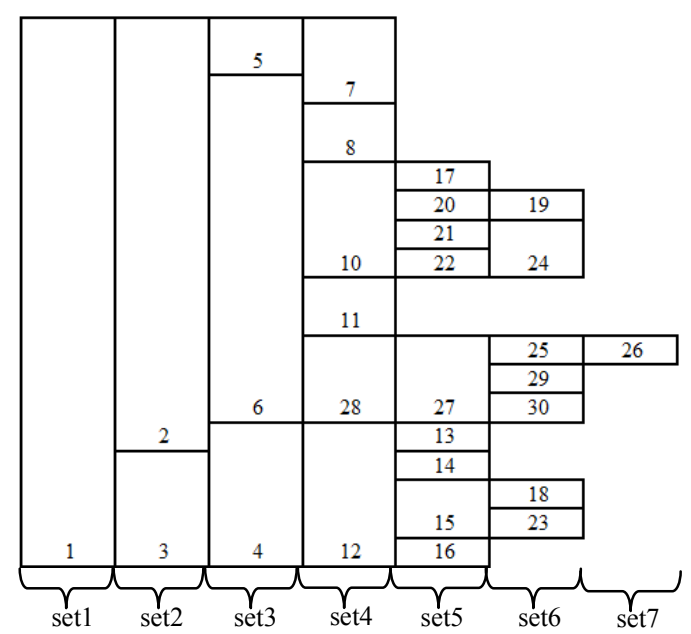

Figure 3. Formulation of the sets for the self-propagating graph beginning at the node corresponding to generator $\mathrm{G}_{1}$

In Fig. 4 node sets are substituted by the corresponding value of the sensitivity $S_{K t h_{k m}}$. The light green color marks the nodes noticeably influencing the stability of generator G1 (meaning that criterion $S_{K t h_{k m}}>S_{K t h}$ min is satisfied), orange is for the first nodes on the path which are not matching the criterion of minimal sensitivity and marked as a dead-end vertexes. Calculation of sensitivities for the nodes lying further along the path is not processed and they are not included in the graph (they are marked with gray color).

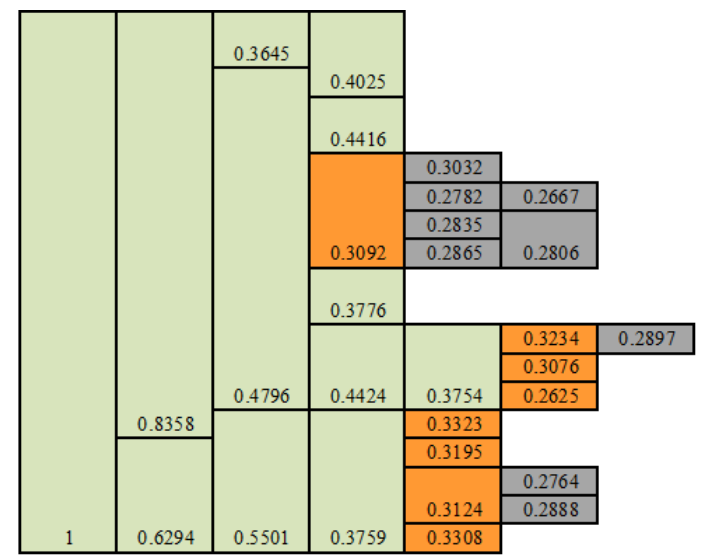

Figure 4. $S_{K t h}$ im the corresponding nodes (Fig. 3) seen by generator $\mathrm{G}_{1}$
As it can be noticed from Fig. 4, the value of sensitivity $S_{K t h_{k m}}$ is lower for all the nodes which path from generator $\mathrm{G}_{1}$ unavoidably passes through the node with sensitivity $S_{K t h_{k m}}<S_{K t h_{\text {min }}}$ and marked as a dead-end. Thus the part of the grid completely isolated from the generator under consideration by nodes marked as dead-end vertexes (Fig. 2) can be a priory excluded from SA as the value of sensitivity for the nodes in this area would invariably be below required minimal preset. This leads to the reduction of the nodes processed through SA from 29 to 20, improving considerably the computational time for the search of optimal countermeasure application nodes.

\section{B. Test of the self-propagating graph method on the 1648 bus US west coast test system ${ }^{1}$}

This Section is considering validation of the selfpropagating graph method on the system containing 1648 nodes among which 313 are generators. For this study the assumption was made that all generators are controlled by Automatic Voltage Regulator (AVR), meaning that the point of constant steady state voltage magnitude $\mathrm{V}_{0}$ is at the terminal of a generator. The admittance matrix, corresponding to the given system was obtained using MATPOWER network simulation packages [8]. According to the methodology described in the Section II the graph, containing nodes which have considerable impact to a given generator stability, should be created. That was done with respect to each generator in the grid, meaning that 313 self-propagating graphs were defined. Variation of admittance only in the load nodes was considered, reflecting the restriction on the re-dispatch of generators. In this study the focus was placed on illustrating the dependency of the self-propagating graph behavior on the value of the parameter $S_{K t h} h_{\min }$. As mentioned in Section II, the value of minimal sensitivity is to be chosen depending on both the topology of the grid and the allocation of control reserves. As neither specific emergency scenario for the grid was considered, nor information about control reserves was available, the comparison analysis for three arbitrarily chosen values of $S_{K t h}$ min was carried out. Those values were picked in order to illustrate the "speed" of sensitivity decrease while increase of the topological distance between a given generator and considered load nodes. In this context the term "speed" defines how deep the self-propagating graph will spread into the grid before it stops due to minimal sensitivity limit. The following three values were considered in this study: $S_{K t h_{\text {min }}}=\left[\begin{array}{lll}0.2 & 0.10 .01\end{array}\right] \cdot S_{K t h}{ }_{\text {max }}, \quad$ which equals to $S_{K t h_{\text {min }}}=\left[\begin{array}{lll}0.2 & 0.10 .01\end{array}\right]$, as $S_{K t h}$ max $=1$ when being normalized. The step of admittance variation applied to each of the load nodes is equal to $0.001 \mathrm{p}$.u. The number of nodes included in the self-propagating graph for each of the generators serves as the parameter reflecting the "speed" of the sensitivity $S_{K t h_{k m}}$ decrease in the grid.

1 The 1648 bus US west coast test system provided in the PSSR E-30.06 is used, http://www.energy.siemens.com/us/en/services/ power-transmission-distribution/power-technologies-international/ software-solutions/pss-e.htm 


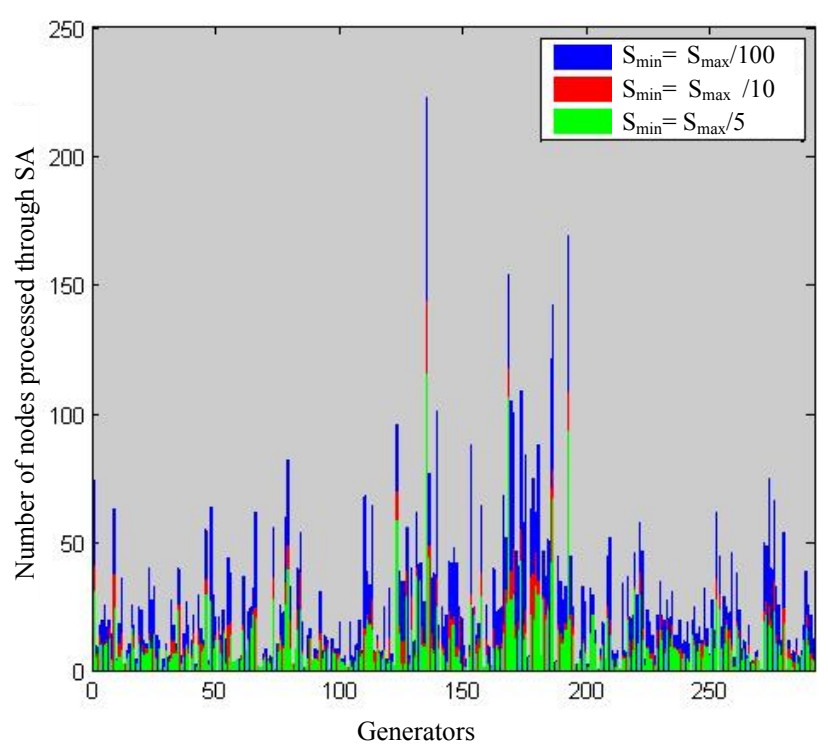

Figure 5. Number of nodes added to the self-propogating graph depending on the chosen value of $S_{K t h}{ }_{\min }$

Fig. 5 demonstrates the joint bar diagram, where the number of nodes included in the self-propagating graph for each generator in the grid when applying different values of $S_{K t h_{\min }}$ is presented. The colors: red, green, blue correspond to the chosen values of minimal sensitivity $S_{K t h} h_{\min }=\left[\begin{array}{lll}0.2 & 0.1\end{array}\right.$ 0.01]. Generators are represented over the x-axis, while the $y$ axis is showing the amount of the load nodes added to the self-propagating graph for the certain generator.

The obtained results demonstrate that even for a low value of minimal sensitivity, equal to 0.01 , the maximum amount of nodes for which the SA should be processed for a given generator is 222 , which is considerably less than total number of load nodes in the grid equal to 1325 . It can be clearly seen that the extent of propagation of the graph inside the grid varies greatly for the different generators within similar value of $S_{K t h} h_{\min }$, which is explained by the fact that the topology of the grid is not homogeneous with the regions of dominating either radial or meshed structure. In meshed topology sensitivity decreases faster this results in smaller number of nodes added to the self-propagating graph.

\section{CONCLUSION}

The presented concept of the self-propagating graph allows considerable reduction of the number of nodes which should be processed through SA, when the search of the optimal node for application of the countermeasure is carried out. For the considered study case of the IEEE 30 bus test system, the initial number of nodes being subject to sensitivity analysis was 29 , while applying the suggested approach this number is reduced to 20 . The case of the 1648 bus US west coast test system has as well demonstrated great reduction in the number of nodes processed through SA - the largest amount of nodes added to the self-propagating graph was 222, while there are 1325 load nodes in the grid. Due to the reduction of the nodes which should be processed through SA, the saving in computational time is gained. Furthermore, it can be noticed that applying the developed method to the larger power systems results in a vast reduction of computational time needed for SA, as less than $20 \%$ of the load nodes are to be considered.

\section{REFERENCES}

[1] M. Glavic and T. Van Cutsem, "Wide area detection of voltage instability from synchronized phasor measurements. Part I: Principle", IEEE Trans. Power Syst., vol. 24, pp.1408-1416, 2009.

[2] M. Glavic and T. Van Cutsem, "Wide area detection of voltage instability from synchronized phasor measurements. Part II: Simulation Results", IEEE Trans. Power Syst., vol. 24, pp.1417-1425 , 2009.

[3] H. Jóhannsson, R. Garcia-Valle, J. T. G. Weckesser, A. H. Nielsen and J. Østergaard, "Real-Time stability assessment based on synchrophasors", IEEE PES Trondheim PowerTech, 2011

[4] H. Jóhannsson, J. Østergaard and A. H. Nielsen, "Identification of critical transmission limits in injection impedance plane", International Journal of Electrical Power \& Energy Systems, vol. 43, no. 1, pp. 433443, Dec. 2012, http://dx.doi.org/10.1016/j.ijepes.2012.05.050

[5] E. Dmitrova, H. Jóhannsson and A.H. Nielsen, "Early prevention of instability - search for optimal grid nodes for applying countermeasures", International Conference on Environment and Electrical Engineering EEEIC, Venice, 2012.

[6] Kruse, Robert L., "Data Structures \& Program Design" (second edition). Englewood Cliffs, New Jersey 07632: Prentice-Hall, Inc. div. of Simon \& Schuster, 1987. ISBN 0-13-195884-4.

[7] Power System Test Archive, available online http://www.ee.washington.edu/research/pstca, Aug.1993.

[8] R. D. Zimmerman, C. E. Murillo-Sánchez, and R. J. Thomas, "MATPOWER: Steady-State Operations, Planning and Analysis Tools for Power Systems Research and Education," Power Systems, IEEE Transactions on, vol. 26, no. 1, pp. 12-19, Feb. 2011. 